УДК 513.88

O. G. STOROZH

\title{
IS IT POSSIBLE TO GIVE A MORE PRECISE FORMULATION OF THE CRITERION OF MAXIMAL ACCRETIVITY FOR ONE EXTENSION OF NONNEGATIVE OPERATOR?
}

\begin{abstract}
O. G. Storozh. Is it possible to give a more precise formulation of the criterion of maximal accretivity for one extension of nonnegative operator?, Mat. Stud. 54 (2020), 107-108.

The conditions being necessary and sufficient for maximal accretivity and maximal nonnegativity of some closed linear operators in Hilbert space are announced. The following problem is proposed: write down these conditions in more convenient form (one of the admissible variants is indicated).
\end{abstract}

Let $H$ be a complex Hilbert space equipped with inner product $(\cdot \mid \cdot)$. The role of the initial object in this communication is played by a closed linear nonnegative operator $L_{0}: H \rightarrow H$ having the domain $D\left(L_{0}\right)$ dense in $H$. Under $L, L_{F}, L_{K}$ we understand its adjoint, hard (Friedrichs), and soft (Neumann-Krein) extensions, respectively. Suppose that a fixed boundary value space $\left(G, \Gamma_{1}, \Gamma_{2}\right)$ of $L_{0}$ and the corresponding Weyl function $M(\lambda)$ are given (we refer a reader to $[1$, p. 256-264] for the details). We keep the following notations: $D(T)$, $\operatorname{ker} T$ are the domain and the kernel of (a linear) operator $T$, respectively; $\mathcal{B}(T)$ is the set of all linear bounded operators $A: G \rightarrow G$ such that $D(A)=G$; for each $A \in \mathcal{B}(T)$ where $G$ is a Hilbert space, $A^{*}$ means the adjont of $A$. The main object of our investigation is the operator $L_{1} \subset L$ such that

$$
D\left(L_{1}\right)=\left\{y \in D(L): A_{1} \Gamma_{1} y+A_{2} \Gamma_{2} y=0\right\},
$$

where $A_{1}, A_{2} \in \mathcal{B}(G)$. We assume below that $L_{0}$ is not positively definite operator, nevertheless

$$
D\left(L_{F}\right)+D\left(L_{K}\right)=D(L), \quad D\left(L_{F}\right) \cap D\left(L_{K}\right)=D\left(L_{0}\right) ;
$$

sequently there exists the strong limit $s-\lim _{\lambda \rightarrow-0} M(\lambda):=M_{0}(\in \mathcal{B}(G))$. Moreover, we suppose that $D\left(L_{F}\right)=\operatorname{ker} \Gamma_{2}$ (the latter suggestion does not lead to the essential loss of generality).

Remind that a linear operator $T: H \rightarrow H$ is said to be an accretive if

$$
\forall y \in D(T) \quad \operatorname{Re}(T y \mid y) \geq 0,
$$

and maximal accretive if, besides, it has no accretive extensions in $H$.

2010 Mathematics Subject Classification: 47A55, 47Gxx.

Keywords: Hilbert space; operator; accretivity.

doi:10.30970/ms.54.1.107-108

(C) O. G. Storozh, 2020 
Theorem 1. $L_{1}$ is a maximal accretive (maximal nonnegative) operator if and only if

i) $A_{1} M_{0} A_{1}^{*}+\operatorname{Re}\left(A_{1} A_{2}^{*}\right) \leq 0 \quad\left(A_{1} M_{0} A_{1}^{*}+A_{1} A_{2}^{*} \leq 0\right)$;

ii) for some (sequently for each) $\lambda<0 \quad \operatorname{ker}\left(A_{1}-A_{2}-A_{1} M(\lambda)\right)=\{0\}$

(compare with [1, p.373-374]).

Problem 1. Is it correct (under the expressed above assumptions) to replace ii) by ii) $\operatorname{ker}\left(A_{1}-A_{2}-A_{1} M_{0}\right)=\{0\}$ ?

In the case when $L_{F}$ is a positively definite operator, it is true.

\section{REFERENCES}

1. Derkach V.A., Malamud M.M. Theory of the extensions of symmetric operators and boundary problems. Works of the Institute of Mathematics of the National Academy of Sciences of Ukraine. Kyiv, 2017. (in Russian)

Faculty of Mechanics and Mathematics Lviv Ivan Franko National University storog@ukr.net 